\title{
Traduire
}

Revue française de la traduction

$231 \mid 2014$

À table !

\section{Retraduire Mark Twain aujourd'hui : entretien avec Bernard Hœpffner}

Propos recueillis par Corinne Wecksteen-Quinio

Corinne Wecksteen-Quinio et Bernard Hœpffner

\section{OpenEdition}

\section{Journals}

Édition électronique

URL : http://journals.openedition.org/traduire/671

DOI : $10.4000 /$ traduire.671

ISSN : 2272-9992

Éditeur

Société française des traducteurs

Édition imprimée

Date de publication : 1 décembre 2014

Pagination : 86-91

ISSN : 0395-773X

\section{Référence électronique}

Corinne Wecksteen-Quinio et Bernard Hœpffner, « Retraduire Mark Twain aujourd'hui : entretien avec Bernard Hœpffner », Traduire [En ligne], 231 | 2014, mis en ligne le 01 décembre 2016, consulté le 03 mai 2019. URL : http://journals.openedition.org/traduire/671 ; DOI : 10.4000/traduire.671 


\section{Retraduire Mark Twain aujourd'hui : entretien avec Bernard Hœpffner}

\section{Propos recueillis par Corinne Wecksteen-Quinio}

Le traducteur Bernard Hœpffner nous a fait le plaisir de nous accorder un entretien(1), au cours duquel nous avons pu l'interroger sur sa pratique traductive, et en particulier sur sa retraduction de Huckleberry Finn, de Mark Twain, parue en septembre 2008 aux éditions Tristram. Nous vous livrons ici l'échange passionnant que nous avons eu : il éclaire des aspects de la traduction parfois inaccessibles et nous permet de cerner au plus près le travail effectué par le traducteur, cet " artisan " qui, s'il n'est pas l'auteur, n'en est pas moins un écrivain, comme le dit si bien Bernard Hœpffner.

J'aimerais commencer par une question relative à Huckleberry Finn, mais qui n'est pas directement liée à sa traduction : que pensez-vous de la décision de la maison d'édition américaine NewSouth de remplacer nigger par slave et Injun par Indian? Diriez-vous que le phénomène du politiquement correct, de la censure, affecte encore la traduction de nos jours?

Cette décision est inadmissible. Cela reflète une notion de la lecture qui est complètement fausse : la lecture, c'est découvrir l'Autre, et refuser le mot nigger tel que Mark Twain l'utilisait, cela veut dire que l'on n'est pas capable de se mettre à la place de quelqu'un qui écrivait en 1860. Ce sont les professeurs qui enseignent Mark Twain aujourd'hui qui sont gênés par l'utilisation de ce mot, pas les lecteurs. Quand j'ai traduit Huck Finn, j'avais décidé au départ que nigger serait traduit par " nègre ". Mais le problème est double, car nigger aujourd'hui est positif. Une femme peut très bien parler de son mari en disant "my nigger". Quelqu'un qui utilise nigger dans ce sens et n'arrive pas à se mettre dans le passé va comprendre autre chose que ce que dit Mark Twain. Beaucoup de mots ont changé de sens, toute la lecture est là.

Les éditeurs ont tendance à faire du " $P C$ " dans un autre sens, pour les livres de jeunesse scandinaves par exemple, en demandant de faire passer l'heure du dîner de $18 \mathrm{~h}$ à $20 \mathrm{~h}$, parce que les enfants français ne comprendraient pas! J'ai le même problème avec le mot dinner en

(1) Entretien réalisé le 18 octobre 2011 à I'Université Charles-de-Gaulle-Lille 3. 
américain, et chez Mark Twain par exemple, qui renvoie au repas du midi : on me dit que les lecteurs ne comprendront pas ; mais si, ils comprendront, car il y a tout le reste, le contexte dans lequel ce mot s'inscrit. Je n'ai jamais eu de problème avec les éditeurs concernant les mots " polack " ou " rital " dans mes traductions de Gilbert Sorrentino, qui est anti-PC, et c'est un vrai plaisir pour le traducteur de pouvoir utiliser tous ces mots-là.

Cela dit, quand j'ai traduit La Prodigieuse Procession et autres charges, qui vient de sortir chez Agone et regroupe des textes politiques de Mark Twain, il y a eu de nombreuses discussions avec la maison d'édition car je voulais inclure un texte sur les Juifs, texte qui était prosémite mais écrit comme on était philosémite en 1890, c'est-à-dire avec l'image du Juif qui gagne beaucoup d'argent, etc. II n'a finalement pas été publié, pour des raisons politiques et pour éviter que le livre dans son ensemble soit attaqué en raison d'un seul texte. J'ai compris ces raisons, mais ce qui me gêne c'est que l'éditeur a pensé, à juste titre peut-être, que les lecteurs n'auraient pas pu transposer leur point de vue à une autre époque.

\section{Qu'est-ce qui a motivé les nouvelles traductions de Tom Sawyer et de Huckleberry Finn?}

II s'agissait d'un projet que je nourrissais depuis plusieurs années. Les éditions Actes Sud l'avaient refusé et il s'avère que la maison d'édition Tristram envisageait également de faire retraduire Mark Twain. Ces deux projets indépendants au départ se sont donc rencontrés, à un moment où l'on commençait à se rendre compte que Twain était un auteur majeur, tout à fait contemporain, et que cela valait la peine de sortir Huckleberry Finn du carcan « littérature de jeunesse " dans lequel il était enfermé.

Vous avez indiqué que pour rendre le mouvement de l'écriture, et en particulier celle de Twain, vous procédiez sans trop de filets, avec un premier jet, suivi de relectures successives. Pouvez-vous expliquer comment vous concevez le travail du traducteur?

Avec la passion, l'expérience, la connaissance, le traducteur peut travailler à toute vitesse et faire des choses sans trop y réfléchir, pour aboutir à des solutions qu'il n'aurait sans doute pas pu trouver par d'autres moyens. Je défends l'idée que le travail du traducteur doit se rapprocher du travail de l'écrivain. II faut cesser d'avoir peur de la critique, du faux sens. Ce que l'on fait, c'est une traduction, ce n'est pas une "version " universitaire, destinée à vérifier un niveau de langue. Le rapport au texte est tout à fait différent. J'attache beaucoup d'importance à ce que j'appellerais l'» intuition de l'écrivain ". J'aime d'ailleurs citer Coleridge qui, dans Biographia Literaria, donne la règle suivante : "Tant que vous n'aurez pas compris l'ignorance d'un écrivain, il faut supposer que vous êtes ignorant de sa compréhension "(2). J'accorde donc une grande importance au premier jet, qui est pour moi très rapide, environ deux semaines pour ce qui est de Huckleberry Finn, un travail sur le rythme, presque une improvisation. Puis je reprends le texte comme si c'était un texte français, afin d'éliminer les anglicismes, qui sont très

(2) Until you understand a writer's ignorance, presume yourself ignorant of his understanding. (S.T. Coleridge, Collected Works of Samuel Taylor Coleridge 7: Biographia Literaria, ed. James Engell and W. Jackson Bate, Princeton: Princeton University Press, 1983, p. 232, traduction de Bernard Hœpffner). 
nombreux chez moi lors de cette phase d'écriture, et d'affiner le rythme. II est également nécessaire de faire des allers-retours avec le texte de départ. En tout, parfois six lectures, et si possible sur papier, pas à l'écran.

Pour le rendu des dialectes de Huckleberry Finn, vous avez indiqué avoir relu Queneau, ainsi que Céline, entre autres : quels éléments de Queneau avez-vous particulièrement retenus?

J'ai surtout retenu les néologismes, avec Zazie dans le métro au départ, ainsi que Les œuvres complètes de Sally Mara, deux ouvrages que j'ai relus au moment où je faisais la traduction de Huckleberry Finn. La façon dont Queneau transpose le langage, qui est tout à fait similaire à ce qu'avait fait Mark Twain un siècle plus tôt, est assez particulière, dans la mesure où il ne s'agit pas de transcrire fidèlement la réalité (personne ne parle comme les personnages de Zazie ou comme ceux de Huck Finn) mais de prendre ce que disent les gens pour élaborer ensuite une langue littéraire à part entière. Cela m'a beaucoup aidé pour le rythme de transposition. Assez étrangement, Queneau, qui était aussi traducteur, a fait cette horrible traduction de Tutuola (L'ivrogne dans la brousse), alors que par ailleurs ses autres traductions (de George du Maurier par exemple) étaient plutôt bonnes. Pour ce qui est des influences, il y a aussi Jacques Roubaud, que j'ai traduit en anglais, et dont le " maître " était Queneau.

Le " polysystème " français ayant déjà eu son Queneau et son Céline, en quoi réside le caractère novateur de vos traductions ? Serait-il lié aux statuts différents qu'occupent l'auteur et le traducteur, et au fait qu'en tant que traducteur vous ayez osé faire ce que l'on n'acceptait auparavant que de la part d'un auteur?

Plutôt que de m'influencer, Queneau m'a " autorisé " à écrire des choses comme "voilatipas ", à prendre des libertés par rapport au carcan que l'on suppose trop facilement être le quotidien du traducteur. En fait, Queneau et Céline ont eu peu d'influence sur la littérature et peu de descendance (contrairement à Twain) car la plupart des écrivains français écrivent encore en " beau français ", à la Julien Gracq. Aussi, quand j'ai traduit Huck Finn, j'avais l'impression de faire œuvre d'auteur, et je veux bien accepter de dire que ceci renvoie à l'hubris du traducteur : ce que j'aimerais, c'est apporter quelques changements à la langue française. Pour moi, la grande époque de la traduction, c'est la Renaissance, car les traducteurs étaient des auteurs. La plupart des œuvres de la Renaissance sont des traductions. En France, les traducteurs n'existent pas en tant que créateurs de langue, et d'ailleurs, il est symptomatique que les dictionnaires français, contrairement aux dictionnaires anglais, ne citent pas d'exemples qui soient des traductions (à moins que les traducteurs ne soient également auteurs, comme Baudelaire), ce qui contribue à renforcer cette idée de l'invisibilité du traducteur(3).

L'universitaire Judith Lavoie proposait d'employer un français créolisé pour rendre la voix de Jim : avez-vous à un moment envisagé d'y recourir ? Vous avez vous-même

(3) Voir l'article de Bernard Hœpffner intitulé «Proxy literature » dans le Times Literary Supplement du 27 mai 2011. 
indiqué qu'un ami caribéen vous avait suggéré d'inventer un parler noir. Quelles en sont les caractéristiques ? Dans quelle mesure pensez-vous avoir réussi à rendre compte du Black English?

Je n'ai pas envisagé d'utiliser un français créolisé. Cela dit, je n'ai pas réussi à rendre compte de la voix de Jim, c'est là le double bind du traducteur : toute solution est critiquable. J'en ai d'ailleurs parlé avec Anne-Laure Tissut, la traductrice de Percival Everett. C'est la raison pour laquelle j'avais toujours hésité à toucher à la littérature du sud des États-Unis, car quoi que l'on fasse, c'est mauvais. J'ai eu droit à des éloges quand est sortie la traduction, mais ce n'était pas très difficile vu les traductions précédentes. J'ai eu recours à un mélange d'inventions verbales, de manipulations, telles que l'élision des $\langle r\rangle$, qui consistent, comme le fait un auteur, à faire croire au lecteur qu'il entend un Noir parler. La grande différence avec les traductions précédentes, c'est que cette représentation artificielle de la langue de Jim est visible tout au long du roman et pas seulement lors de la première phrase.

On remarque que la voix de Jim n'est pas traduite de façon totalement homogène dans l'ensemble de Huck Finn (l'élision du $<r>$ n'est pas systématique par exemple, ou encore on trouve à la fois " ils étaient " et " y zétaient " dans la bouche de Jim). S'agit-il d'un choix délibéré ? Avez-vous reçu des consignes de l'éditeur ou était-ce une volonté de procéder par touches impressionnistes pour ne pas donner un côté trop mécanique au texte, en raison d'une résistance du français par rapport à une typographie " déviante " ?

En effet, il s'agit d'éviter le côté mécanique. J'enlève les $\langle r\rangle$, mais pas toujours, pas partout. Quand on écoute des Noirs, il y a certes une élimination $d u<r\rangle$, mais je ne veux pas faire $d u$ "petit nègre ", ce ne sont pas des Africains, ce sont des Noirs des États-Unis. L'élision du $<r>$ intervient souvent en finale, pour l'aspect visuel, car il s'agit encore et toujours d'une entreprise de manipulation du lecteur, même si cela ne correspond pas à la façon dont parlent effectivement les Noirs. C'est là que le premier jet est important : on se lance, puis on revient en arrière et on utilise un français un tout petit peu plus normal que ce qui figurait dans le premier jet. C'est une question de rythme, il s'agit de "mâchonner " la phrase jusqu'à ce que ça passe, même si ce n'est pas logique ou explicable.

Comment justifiez-vous le fait d'utiliser des termes anachroniques par rapport à l'époque où se situe le roman (on a un mélange de termes à la fois anciens, comme "victailles ", et très contemporains ou néologiques, comme "stylé ") ?

La traduction est de toute façon anachronique car j'écris dans un français d'aujourd'hui. Cela fait partie de la manipulation de l'écrivain, qui consiste à faire croire à son lecteur qu'il lit un livre dont l'action se passe aux États-Unis en 1850, mais c'est là tout le jeu de la littérature. Tout est anachronique, mais j'ai aussi inséré ici et là à dessein des termes anciens, afin de montrer au lecteur qu'il lisait un texte ancien. Je ne sais plus si " victailles " est véritablement une invention ou s'il s'agit d'un terme que j'avais lu quelque part, auquel cas il s'agit de réinventer quelque chose qui existait déjà. Par ailleurs, "stylé ", que j'utilise, existait déjà à l'époque mais avec un sens différent de celui dans lequel il est utilisé par les jeunes aujourd'hui. 
Vous revendiquez le fait que le texte traduit comporte une part d'étrangeté et qu'il puisse être plus difficile à lire qu'un ouvrage écrit directement dans sa propre langue. Jusqu'où le traducteur peut-il aller dans la transgression, dans la violence faite à la langue, dans la fécondation du " Propre par la médiation de l'Étranger ", dirait Berman ? Doit-il se fixer des limites?

La traduction doit essayer d'être aussi proche que possible du texte de départ. II y a d'ailleurs toute une époque où je disais être complètement sourciste. Je n'y crois plus beaucoup maintenant car je m'aperçois, à la lumière des écrits de Meschonnic, que je peux être tout aussi infidèle qu'un cibliste par moments. Idéalement, j'aimerais que tous les aspects culturels, étymologiques du texte de départ ressortent dans le texte d'arrivée, mais il faut que le texte fasse la même taille. II faut donc accepter dès le départ qu'on ne pourra pas faire un certain nombre de choses, que des concepts, des connotations disparaîtront et que la traduction est inférieure à l'original. Mais il faut aussi faire violence au français quand les originaux font violence à leur langue, et la latitude du traducteur dépendra également de l'éditeur. Avec Tristram, il y a un espace de liberté car ils me font confiance, parce que la discussion est possible.

Avez-vous parfois des velléités d'écriture ? Pensez-vous un jour, à l'instar du traducteur Brice Matthieussent par exemple, ressentir le besoin d'écrire un roman par/pour vousmême et non " by proxy ", pour reprendre en partie le sous-titre de l'un de vos articles?

J'ai toujours écrit, que ce soit des textes sur la littérature (Guy Davenport, Robert Burton), sur la traduction, ou encore des préfaces ou des postfaces. De plus, tout traducteur est un écrivain, et la traduction prend beaucoup de temps, ce qui comble en grande partie ce désir d'écriture. Cela dit, je suis en train d'écrire un livre qui s'intitule Portrait du traducteur en escroc. II tient à la fois du roman et de l'essai car il contient certains éléments de textes que j'ai écrits, comme Portrait of the Translator as a Chameleon par exemple. Cela dit, ce n'est pas un roman sur moi, même s'il y a une partie autobiographique évidemment.

Pour conclure cet entretien, quelles autres œuvres américaines du XIXe siècle avez-vous traduites?

J'ai traduit plusieurs œuvres de Herman Melville : Bartleby, Trois contes doubles et Les îles enchantées. Par ailleurs, je travaille à la traduction de la volumineuse autobiographie inédite de Mark Twain, dont le premier volume est paru en anglais aux États-Unis fin 2010, soit cent ans après la mort de l'auteur, qui avait imposé ce délai avant toute publication. Le premier tome paraîtra, toujours chez Tristram, en 2012(4), les deux suivants sortiront en 2014 et en 2016.

b.hoepffner@orange.fr - corinne.wecksteen@univ-artois.fr

(4) Ouvrage paru depuis la réalisation de cet entretien : Twain Mark, 2012, L'autobiographie de Mark Twain : une histoire américaine, Auch, Tristam, traduit par Bernard Hœpffner. 
Né en 1946, Bernard Hœpffner a passé son enfance en Allemagne et son adolescence en France. Il a ensuite vécu en Angleterre où il était restaurateur d'objets d'Extrême-Orient, puis aux Canaries comme agriculteur. De retour en France en 2013, après six ans aux Pays-Bas et en Belgique, il vit à présent dans la Drôme. Il écrit et traduit depuis longtemps et, depuis 1988, se consacre exclusivement à l'écriture et à la traduction (anglais/français et français/anglais) Ce traducteur pluriel par nature possède donc de multiples facettes et plusieurs casquettes : traduction, écriture d'ouvrages (Guy Davenport, L'Utopie localisée, Belin, 1998), d'articles de réflexion sur le travail de traduction (cf. par exemple "L'ombilicalité du traducteur ", paru dans la revue Palimpsestes, $\left.n^{\circ} 23,2010\right)$. II se revendique volontiers comme un " artisan" et compare souvent le traducteur à un caméléon (cf. son article "Portrait of the Translator as a Chameleon ", paru dans Through Other Eyes: The Translation of Anglophone Literature in Europe, Ed. R. Trim \& S. Alatorre, Cambridge Scholars Publishing, 2007), voire à un "escroc ". Ses traductions couvrent des genres et des époques variés : il a traduit aussi bien des romans et des nouvelles que de la poésie ou des essais; il est à l'aise avec les textes classiques comme avec la littérature contemporaine. On trouvera ainsi à son palmarès l'Anatomie de la mélancolie de Robert Burton (XVIIe siècle), mais aussi la littérature expérimentale, avec des auteurs comme Robert Coover, Toby Olson ou encore Gilbert Sorrentino, un de ses écrivains de prédilection. Ses retraductions de Mark Twain (Les Aventures de Tom Sawyer et Aventures de Huckleberry Finn, Editions Tristram, 2008) ont reçu l'approbation unanime de la critique.

\section{http://wvorg.free.fr/hoepffner/spip/spip.php?article1}

Corinne Wecksteen-Quinio est agrégée d'anglais, Maitre de Conférences en traduction/traductologie (anglais - français) à l'Université d'Artois (France), membre du centre de recherches "Textes et Cultures " et auteur en 2005 d'une thèse de doctorat en traductologie intitulée "Traduction et connotation ", sous la direction de Michel Ballard. Elle a consacré deux articles à l'étude de la retraduction de Huckleberry Finn par Bernard Hœeffner : "La retraduction de Huckleberry Finn : Huck a-t-il (enfin) trouvé sa voix ? ", Meta, Vol. 56, $n^{\circ}$ 3, septembre 2011, p. 468-492 et "De Janus à Ménechme : portrait du traducteur en agent double ", in Mariaule Michaël et Corinne Wecksteen (éds), Le double en traduction ou l'(impossible ?) entre-deux (Vol. 1), Arras, Artois Presses Université, 2011, collection "Traductologie ", p. 29-48. Ses recherches traductologiques portent, entre autres, sur la connotation, la variabilité du sens, l'ambiguité et l'implicite, mais aussi sur le lien entre traduction et écriture ainsi que sur la didactique de la traduction. Elle a publié plusieurs articles sur ces thèmes, est co-auteur, avec Michel Ballard, d'un ouvrage sur les faux amis (Ellipses) et a collaboré à l'élaboration d'un manuel de traduction anglais-français mettant en avant la traductologie et le commentaire de traduction (La traduction anglais-français, Manuel de traductologie pratique, Lefebvre-Scodeller Cindy, Michaël Mariaule et Corinne Wecksteen-Quinio, Bruxelles/Louvain-la-Neuve, Éditions De Boeck, collection "Traductologie ", à paraître en 2015). 